Terr. Atmos. Ocean. Sci., Vol. 18, No. 1, 31-53, March 2007

\title{
Dispersion-Correction Finite Difference Model for Simulation of Transoceanic Tsunamis
}

\author{
Sung Bum Yoon ${ }^{1, *}$, Chae Ho Lim ${ }^{1}$, and Junwoo Choi ${ }^{1}$ \\ (Manuscript received 19 September 2006, in final form 20 November 2006)
}

\begin{abstract}
A finite difference numerical model, which can correctly consider dispersion effect of waves over a slowly varying water depth, is developed for the simulation of tsunami propagation. The present model employs a linear Boussinesq-type wave equation that can be solved more easily than typical Boussinesq equations. In the present model numerical dispersion is minimized by controlling the dispersion-correction parameter determined by the time step, grid size and local water depth. In order to examine the applicability of the present model to dispersive waves, the propagation of tsunamis is simulated for an initial water surface displacement of Gaussian shape for the cases of several constant water depths and a submerged circular shoal. The numerical results are compared with analytical solutions or numerical solutions of linearized Boussinesq equations. The comparisons show that satisfactory agreement is obtained.
\end{abstract}

(Key words: Tsunamis, Boussinesq equations, Dispersion-correction, Finite difference)

\section{INTRODUCTION}

Tsunamis are ocean water surface waves generated by undersea earthquakes, landslides, volcanic eruptions or even meteoric impact on water surfaces. A tsunami travels in all directions from the source region where the water surface is disturbed. The tsunamis grow as they approach shallow water and smash onto the shore. This may cause serious damage in coastal areas. In order to reduce such damage, a proper monitoring, forecasting and warning system needs to be established. Consequently, we need to fully understand mechanisms such as generation, propagation, and inundation of tsunamis and to predict possible quantities such as arrival times and run-up heights of a tsunami event. Thus, the development of an accurate and

\footnotetext{
${ }^{1}$ Department of Civil and Environmental Engineering, Hanyang University, Ansan, Korea

* Corresponding author address: Prof. Sung Bum Yoon, Department of Civil and Environmental Engineering, Hanyang University, Ansan, Korea; E-mail: sbyoon@hanyang.ac.kr
} 
efficient numerical model for computing all aspects of tsunami propagation is indispensable.

When tsunamis are generated by an earthquake, they have a shape of solitary wave with a long crest. After they propagate a long distance from the source area over deep oceans, tsunamis evolve into a train of waves due to wave dispersion effect. When the source area is narrow, the dispersion effect plays an important role in the deformation of tsunamis; this is also the case in relatively shallow water areas. Boussinesq equations are one of the best choices for governing equations to construct numerical models for far-field tsunamis as they can take into account the dispersion effects. However, the Boussinesq numerical models, such as FUNWAVE (Kirby et al. 1998) and COULWAVE (Lynett and Liu 2004; Hsiao et al. 2005), require a small mesh size to suppress numerical dispersion errors. This consumes huge amounts of computer resources due to the implicit nature of the solution technique to deal with dispersion terms. Thus, the Boussinesq model is not preferred for the simulation of the far-field tsunamis, and shallowwater equations are generally employed instead.

Considerable efforts to construct a numerical model for the simulation of tsunamis have been made. For example, finite difference models were developed by Hwang and Divoky (1970), Goto and Shuto (1983), Kowalik and Murty (1984), and Mader and Curtis (1991). Most of the existing numerical models are based on the shallow-water equations and have been successfully applied to near-field tsunamis, which have little wave dispersion effect. However, distant tsunamis generated far from the region of interest are mainly transformed due to the accumulation of dispersion effects during propagation. Thus, numerical models based on the shallow-water equations will suffer from a lack of accuracy inherent in the models for the simulation of distant tsunamis.

Imamura et al. (1988) presented a finite difference model for the simulation of transoceanic propagation of tsunamis. The model solves the shallow-water equations using a leap-frog scheme. The physical dispersion is compensated by the numerical dispersion introduced by the truncation error of the numerical scheme. This can be done only if the grid size is appropriately selected for the given water depth and the time step satisfying the criterion proposed by Imamura et al. (1988). Cho (1995) improved the numerical model of Imamura et al. (1988) for tsunamis obliquely propagating to the principal axes of computational grids. However, strictly speaking, those models have no capability to consider the dispersion effect of waves in practical application. The use of numerical models developed by Imamura et al. (1988) and Cho (1995) is limited to the case of constant water depth.

Yoon (2002) developed a new finite difference scheme that uses a uniform grid, but the actual computations are made on a hidden grid of variable size determined from the conditions proposed by Imamura et al. (1988). This model satisfies local dispersion relationships of waves for a slowly varying topography. However, the accuracy of the model degrades when applied to short waves due to numerical errors associated with the interpolation of quantities at hidden grid points.

The primary objective of this study is to develop a highly efficient and relatively accurate two-dimensional finite difference model to simulate the propagation of distant tsunamis over varying topography. The present finite difference model is based on a simplified linear Boussinesq wave equation. A new dispersion-correction scheme instead of adjusting the grid size is employed. The dispersion-correction scheme developed in this study can properly cap- 
ture the dispersion effect by getting rid of the numerical dispersion error arising from the numerical scheme and providing the physical dispersion by controlling the parameters determined from the given time step, grid size and local water depth.

After the tsunamis travel a long distance from the generation region, they experience shoaling and run-up due to decreasing water depth over the continental shelf. Nonlinearity plays a significant role in the transformation of tsunami waves in this region. Moreover, topographic changes are no longer slow. Thus, the accuracy of the present linear far-field model developed for slowly varying topography becomes degraded, and the present model cannot be applied. These difficulties can be solved by nesting the near-field model such as full Boussinesq equation model or shallow water equation model with a finer mesh system to the present transoceanic model as described in Yoon (2002).

\section{GOVERNING EQUATIONS}

For tsunamis of transoceanic propagation nonlinearity of waves can be neglected since free surface displacement against water depth is small. The accuracy of the numerical simulation of a given tsunami can be improved by proper consideration of the dispersion effects of the tsunami. The following linear Boussinesq equations can be used as governing equations for far-field tsunamis:

$$
\begin{aligned}
& \frac{\partial \zeta}{\partial t}+\frac{\partial P}{\partial x}+\frac{\partial Q}{\partial y}=0 \\
& \frac{\partial P}{\partial t}+g h \frac{\partial \zeta}{\partial x}=\frac{h^{2}}{2} \frac{\partial}{\partial x}\left[\frac{\partial}{\partial x}\left(\frac{\partial P}{\partial t}\right)+\frac{\partial}{\partial y}\left(\frac{\partial Q}{\partial t}\right)\right]-\frac{h^{3}}{6} \frac{\partial}{\partial x}\left[\frac{\partial^{2}}{\partial t \partial x}\left(\frac{P}{h}\right)+\frac{\partial^{2}}{\partial t \partial y}\left(\frac{Q}{h}\right)\right], \\
& \frac{\partial Q}{\partial t}+g h \frac{\partial \zeta}{\partial y}=\frac{h^{2}}{2} \frac{\partial}{\partial y}\left[\frac{\partial}{\partial x}\left(\frac{\partial P}{\partial t}\right)+\frac{\partial}{\partial y}\left(\frac{\partial Q}{\partial t}\right)\right]-\frac{h^{3}}{6} \frac{\partial}{\partial y}\left[\frac{\partial^{2}}{\partial t \partial x}\left(\frac{P}{h}\right)+\frac{\partial^{2}}{\partial t \partial y}\left(\frac{Q}{h}\right)\right\rfloor,
\end{aligned}
$$

where $\zeta$ represents the free surface elevation from still water level (m), $P$ and $Q$ are the depthintegrated volume fluxes $\left(\mathrm{m}^{2} \mathrm{~s}^{-1}\right)$ in the $x$ and $y$ directions, respectively. $g$ is the acceleration of gravity $\left(\mathrm{m} \mathrm{s}^{-2}\right)$, and $h$ is the water depth $(\mathrm{m})$.

The equations of motion (2) and (3) include the dispersion terms in the right-hand side. These dispersion terms cause numerical difficulties in practice because of the mixed form of differentiations with respect to both time and space. Thus, an implicit scheme is generally employed. Moreover, they should be solved using fine grids to reduce numerical errors. Consequently, the numerical model requires a significant computational effort due to a huge computer memory space requirement and an excessive execution time to solve Boussinesq equations. 
To develop an efficient and relatively accurate numerical model for the propagation of dispersive tsunamis over slowly varying topography a linear Boussinesq-type wave equation for variable water depth is first derived from the linear Boussinesq equations (1) (3) as:

$$
\begin{aligned}
& \frac{\partial^{2} \zeta}{\partial t^{2}}-\left\lfloor\frac{\partial}{\partial x}\left(g h \frac{\partial \zeta}{\partial x}\right)+\frac{\partial}{\partial y}\left(g h \frac{\partial \zeta}{\partial y}\right) \mid\right. \\
& -\frac{h^{2}}{3}\left[\frac{\partial^{2}}{\partial x^{2}}\left\{\frac{\partial}{\partial x}\left(g h \frac{\partial \zeta}{\partial x}\right)+\frac{\partial}{\partial y}\left(g h \frac{\partial \zeta}{\partial y}\right)\right\}+\frac{\partial^{2}}{\partial y^{2}}\left\{\frac{\partial}{\partial x}\left(g h \frac{\partial \zeta}{\partial x}\right)+\frac{\partial}{\partial y}\left(g h \frac{\partial \zeta}{\partial y}\right)\right]\right\rfloor=0,
\end{aligned}
$$

where the water depth is assumed to be slowly varying in the computational domain. (4) can be split into two first-order partial differential equations in time as:

$$
\begin{aligned}
& \frac{\partial v}{\partial t}=g\left(\frac{\partial h}{\partial x} \frac{\partial \zeta}{\partial x}+\frac{\partial h}{\partial y} \frac{\partial \zeta}{\partial y}\right)+g h\left(\frac{\partial^{2} \zeta}{\partial x^{2}}+\frac{\partial^{2} \zeta}{\partial y^{2}}\right), \\
& \frac{\partial \zeta}{\partial t}=v+\frac{h^{2}}{3}\left(\frac{\partial^{2} v}{\partial x^{2}}+\frac{\partial^{2} v}{\partial y^{2}}\right),
\end{aligned}
$$

where $v$ denotes an auxiliary variable introduced for computational convenience. The solution of (5) and (6) is identical with that of (4). To simulate the propagation of distant tsunamis over a slowly varying topography with improved wave dispersion effect, the new governing equations (5) and (6) can be solved by using a relatively simple explicit numerical scheme.

\section{DISPERSION-CORRECTION SCHEME}

In this section a finite difference numerical scheme to solve (5) and (6) will be first developed for the case of uniform water depth. Then, it will be extended to the case of slowly varying topography.

\subsection{Determination of Dispersion-Correction Parameter}

The linear Boussinesq wave equation (4) can be reduced for the case of uniform water depth under the assumption of long waves (i.e., $k h<\pi 10^{-1}$ ) to the following linear Boussinesq wave equation:

$$
\frac{\partial^{2} \zeta}{\partial t^{2}}-C_{0}^{2}\left(\frac{\partial^{2} \zeta}{\partial x^{2}}+\frac{\partial^{2} \zeta}{\partial y^{2}}\right)-\frac{C_{0}^{2} h^{2}}{3}\left(\frac{\partial^{4} \zeta}{\partial x^{4}}+2 \frac{\partial^{4} \zeta}{\partial x^{2} \partial y^{2}}+\frac{\partial^{4} \zeta}{\partial y^{4}}\right)=O\left(k^{6} h^{6}\right)
$$


where $k$ in the truncated error term denotes the wave number $\left(\mathrm{rad} \mathrm{m}^{-1}\right)$ of uniform waves, and $C_{0}(=\sqrt{g h})$ is the phase speed of waves. The $3^{\text {rd }}$ group of terms in (7) represents the physical dispersion of waves.

On the other hand, Krenk (2001) proposed a modified wave equation (8) by adding an artificial dispersion term to a two-dimensional wave equation to eliminate the numerical dispersion errors as:

$$
\frac{\partial^{2} \zeta}{\partial t^{2}}-C_{0}^{2}\left(\frac{\partial^{2} \zeta}{\partial x^{2}}+\frac{\partial^{2} \zeta}{\partial y^{2}}\right)+\gamma C_{0}^{2} \Delta x^{2}\left(\frac{\partial^{4} \zeta}{\partial x^{4}}+2 \frac{\partial^{4} \zeta}{\partial x^{2} \partial y^{2}}+\frac{\partial^{4} \zeta}{\partial y^{4}}\right)=0
$$

where $\gamma$ is a dispersion-correction parameter introduced to eliminate the numerical dispersion errors caused by the explicit finite difference scheme to solve the original wave equation. The original wave equation is recovered if $\gamma=0.0$. The wave equation (8) proposed by Krenk (2001) is similar to the linear Boussinesq wave equation (7). In the present study, the dispersion-correction parameter $\gamma$ will be chosen to minimize the numerical dispersion error and also to provide the physical dispersion of waves. As a result the numerical solution of (8) will be very close to the solution of (7).

The equation (8) is split into two first-order partial differential equations in time as:

$$
\begin{aligned}
& \frac{\partial v}{\partial t}=C_{0}^{2}\left(\frac{\partial^{2} \zeta}{\partial x^{2}}+\frac{\partial^{2} \zeta}{\partial y^{2}}\right), \\
& \frac{\partial \zeta}{\partial t}=v-\gamma \Delta x^{2}\left(\frac{\partial^{2} v}{\partial x^{2}}+\frac{\partial^{2} v}{\partial y^{2}}\right),
\end{aligned}
$$

To obtain an explicit finite difference representation of the linear Boussinesq wave equations (9) and (10) a leap-frog difference approximation is used for the time derivatives, and a second-order central difference approximation is used for the spatial derivatives. The finite difference approximation of (9) and (10) can be written as:

$$
\begin{aligned}
& \frac{v_{i, j}^{n+1 / 2}-v_{i, j}^{n-1 / 2}}{\Delta t}-C_{0}^{2}\left(\frac{\zeta_{i+1, j}^{n}-2 \zeta_{i, j}^{n}+\zeta_{i-1, j}^{n}}{\Delta x^{2}}+\frac{\zeta_{i, j+1}^{n}-2 \zeta_{i, j}^{n}+\zeta_{i, j-1}^{n}}{\Delta y^{2}}\right)=0, \\
& \frac{\zeta_{i, j}^{n+1}-\zeta_{i, j}^{n}}{\Delta t}-v_{i, j}^{n+1 / 2}+\gamma \Delta x^{2}\left(\frac{v_{i+1, j}^{n+1 / 2}-2 v_{i, j}^{n+1 / 2}+v_{i-1, j}^{n+1 / 2}}{\Delta x^{2}}+\frac{v_{i, j+1}^{n+1 / 2}-2 v_{i, j}^{n+1 / 2}+v_{i, j-1}^{n+1 / 2}}{\Delta y^{2}}\right)=0,
\end{aligned}
$$

where indices $i$ (and $j$ ) and $n$ represent a spatial grid point and a time level, respectively, as shown in Fig. 1. $\Delta x$ and $\Delta y$ are the spatial grid sizes, and $\Delta t$ denotes the time step. 

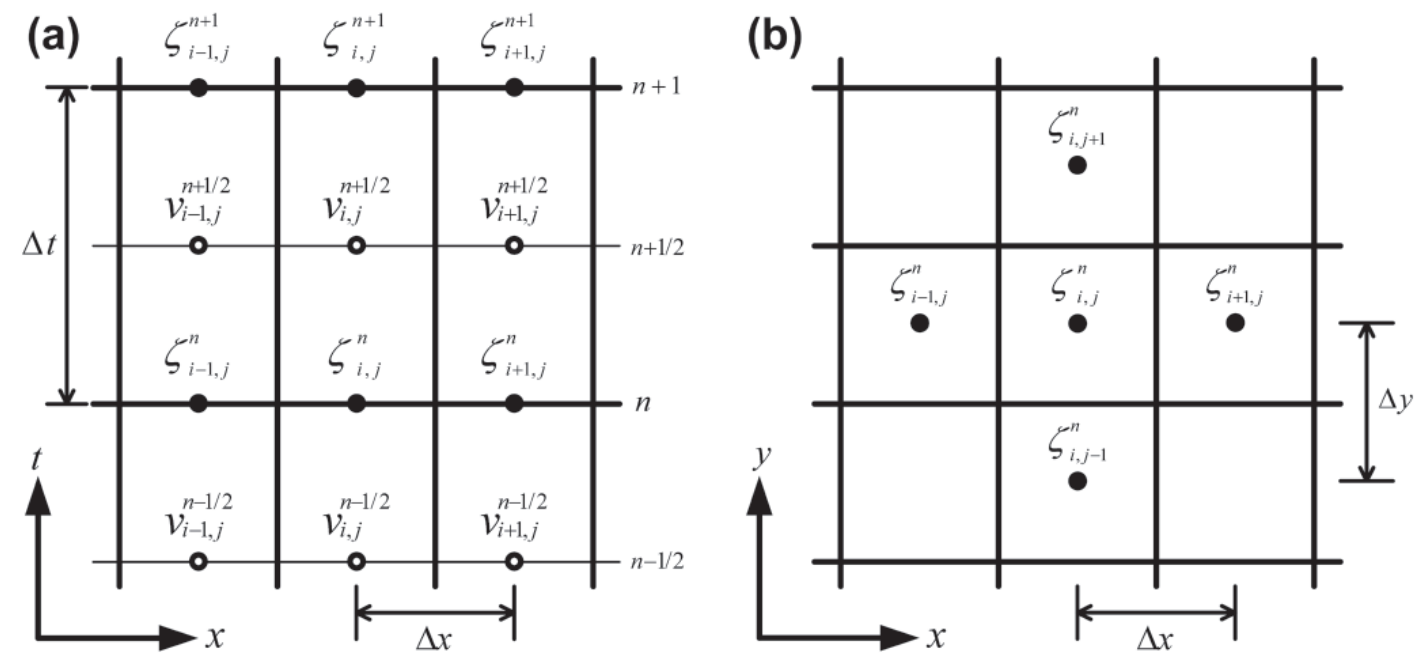

Fig. 1. Sketch of the arrangement of variables and grid points. (a) Grid system in time; (b) Grid system in space.

This finite difference scheme gives numerical dispersion errors. The effects of the numerical dispersion can be clearly understood by deriving the following modified equation of (11) and (12) through the expansion of each equation using the Taylor series as:

$$
\begin{aligned}
& \frac{\partial^{2} \zeta}{\partial t^{2}}-C_{0}^{2}\left(\frac{\partial^{2} \zeta}{\partial x^{2}}+\frac{\partial^{2} \zeta}{\partial y^{2}}\right)-\frac{1}{12} C_{0}^{2} \Delta x^{2}\left(1-12 \gamma-C_{r}^{2}\right)\left(\frac{\partial^{4} \zeta}{\partial x^{4}}+2 \frac{\partial^{4} \zeta}{\partial x^{2} \partial y^{2}}+\frac{\partial^{4} \zeta}{\partial y^{4}}\right) \\
& +\frac{1}{6} C_{0}^{2} \Delta x^{2} \frac{\partial^{4} \zeta}{\partial x^{2} \partial y^{2}}=O\left(\Delta x^{4}, \Delta x^{2} \Delta t^{2}, \Delta x \Delta t^{3}, \Delta t^{4}\right),
\end{aligned}
$$

where $C_{r}\left(=\mathrm{C}_{0} \Delta t / \Delta x\right)$ represents the Courant number. In the derivation of (13) from (11) and (12), it is assumed that the water depth $h$ is uniform, and $\Delta x=\Delta y$. The $3^{\text {rd }}$ and $4^{\text {th }}$ groups of terms in the left-hand side of (13) are the truncation errors due to numerical discretization. The truncation errors of (13) are so called numerical dispersion that affects the phase speed of wave propagation.

Krenk (2001) insisted that the truncation errors can be eliminated if the dispersion-correction parameter $\gamma$ is equal to $1 / 12$ and the dispersion free solution for the wave equation can be obtained. However, the $3^{\text {rd }}$ group of terms can be eliminated only in the case of an infinitesimally small time step. To get rid of the dispersion error associated with the $3^{\text {rd }}$ group of terms in (13) completely, the dispersion-correction parameter $\gamma$ should be $\left(1-C_{r}^{2}\right) / 12$. On top of 
this, the $4^{\text {th }}$ group of terms introduces an additional dispersion error for the waves propagating obliquely to the principal axes of the grid system and should also be eliminated.

On the other hand, Imamura et al. (1988) proposed a leap-frog finite difference model to simulate the propagation of distant tsunamis based on the linear shallow-water equations. Imamura et al. (1988) found that the numerical dispersion inherent in the leap-frog scheme mimics the physical dispersion of (4) in $x$ and $y$ directions for uniform water depth if the grid size is chosen to satisfy the following criterion:

$$
\Delta x_{\mathrm{Im}}=\sqrt{4 \mathrm{~h}^{2}+g h \Delta t^{2}} .
$$

However, the dispersion-correction in the diagonal direction is incomplete. As a result, the conventional model developed by Imamura et al. (1988) gives a less dispersive solution in the diagonal direction. Cho (1995) made an improvement to achieve a uniform dispersioncorrection in every direction by adopting the idea of Abbott et al. (1981).

In the present study the finite difference representation (11) is reformulated based on the grid system shown in Fig. 2 to achieve an orientation-free dispersion-correction as proposed by Abbott et al. (1981).

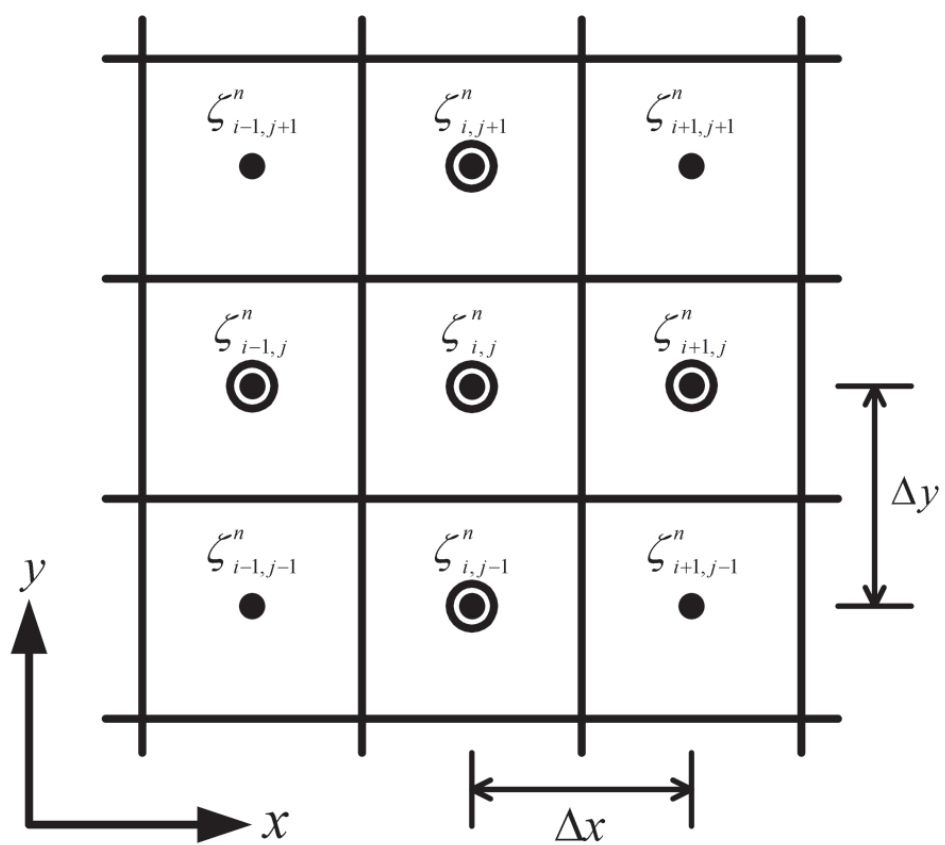

Fig. 2. Sketch of the arrangement of variables for dispersion-correction in diagonal direction. 
The following finite difference approximation of (9) is obtained:

$$
\begin{aligned}
\frac{v_{i, j}^{n+1 / 2}-v_{i, j}^{n-1 / 2}}{\Delta t} & -g h(1-\alpha)\left(\frac{\zeta_{i+1, j}^{n}-2 \zeta_{i, j}^{n}+\zeta_{i-1, j}^{n}}{\Delta x^{2}}+\frac{\zeta_{i, j+1}^{n}-2 \zeta_{i, j}^{n}+\zeta_{i, j-1}^{n}}{\Delta y^{2}}\right) \\
& +\frac{\alpha}{2}\left(\frac{\zeta_{i+1, j-1}^{n}-2 \zeta_{i, j-1}^{n}+\zeta_{i-1, j-1}^{n}}{\Delta x^{2}}+\frac{\zeta_{i+1, j+1}^{n}-2 \zeta_{i, j+1}^{n}+\zeta_{i-1, j+1}^{n}}{\Delta x^{2}}\right) \\
& +\frac{\alpha}{2}\left(\frac{\zeta_{i-1, j+1}^{n}-2 \zeta_{i-1, j}^{n}+\zeta_{i-1, j-1}^{n}}{\Delta y^{2}}+\frac{\zeta_{i+1, j+1}^{n}-2 \zeta_{i+1, j}^{n}+\zeta_{i+1, j-1}^{n}}{\Delta y^{2}}\right) \mid=0,
\end{aligned}
$$

where $\alpha$ is a weighting factor to be determined later. The modified equation of the proposed finite difference equations (15) and (12) is derived, and the result is presented here as:

$$
\begin{aligned}
& \frac{\partial^{2} \zeta}{\partial t^{2}}-C_{0}^{2}\left(\frac{\partial^{2} \zeta}{\partial x^{2}}+\frac{\partial^{2} \zeta}{\partial y^{2}}\right)-\frac{1}{12} C_{0}^{2} \Delta x^{2}\left(1-12 \gamma-C_{r}^{2}\right)\left(\frac{\partial^{4} \zeta}{\partial x^{4}}+2 \frac{\partial^{4} \zeta}{\partial x^{2} \partial y^{2}}+\frac{\partial^{4} \zeta}{\partial y^{4}}\right) \\
& -\left(\alpha-\frac{1}{6}\right) C_{0}^{2} \Delta x^{2} \frac{\partial^{4} \zeta}{\partial x^{2} \partial y^{2}}=O\left(\Delta x^{4}, \Delta x^{3} \Delta t, \Delta x^{2} \Delta t^{2}, \Delta t^{4}\right) .
\end{aligned}
$$

To get an orientation-free scheme the $4^{\text {th }}$ term in (16) is first eliminated by setting $\alpha=1 / 6$. Next, the dispersion-correction parameter $\gamma$ is determined here. If the coefficient of the $3^{\text {rd }}$ group of terms in the linear Boussinesq wave equation (7) is set to be equal to that of the modified wave equation (16), the two equations become identical. Thus, the dispersion-correction parameter $\gamma$ is determined as:

$$
\gamma=\frac{\Delta x^{2}-\left(4 \mathrm{~h}^{2}+g h \Delta t^{2}\right)}{12 \Delta x^{2}}=\frac{1}{12}\left[1-\left(\frac{\Delta x_{\mathrm{Im}}}{\Delta x}\right)^{2}\right] .
$$

If the dispersion-correction parameter $\gamma$ calculated by (17) for given local water depth, spatial grid size and time step is used, a considerably accurate solution can be achieved for the linear Boussinesq wave equation (7). Thus, the selection of grid size $\Delta x$ is free from condition (14) of Imamura et al. (1988). This means that the present dispersion-correction scheme is much more flexible in the selection of grid size than the conventional scheme. We remark here that when the uniform grid size $\Delta x$ is selected for constant water depth to satisfy condition (14), then $\gamma$ becomes 0.0 and the present scheme is reduced to the numerical scheme of Imamura et al. (1988). This implies that the scheme developed by Imamura et al. (1988) is a special case of the present scheme for constant water depth. 


\subsection{Stability Analysis}

The stability analysis of the self-adjusting dispersion-correction numerical scheme is conducted under the condition of the uniform waves over constant water depth following the Fourier stability analysis of Lapidus and Pinder (1982). By substituting the Fourier components for each time step and spatial grid point into the finite difference equations (15) and (12), the amplification factor $G$ can be obtained. The procedure is straightforward but lengthy. Thus, only the final result is presented here as:

$$
G=\delta \pm \sqrt{\delta^{2}-1}
$$

where

$$
\begin{aligned}
& \delta=1+C_{r}^{2}\left(\delta_{1}-\gamma \delta_{2}\right), \\
& \delta_{1}=\frac{1}{6} \cos (2 k \Delta x)+\frac{4}{3} \cos (k \Delta x)-\frac{3}{2}, \\
& \delta_{2}=\frac{1}{3} \cos (3 k \Delta x)+2 \cos (2 k \Delta x)-11 \cos (k \Delta x)+\frac{26}{3},
\end{aligned}
$$

where $\Delta x=\Delta y$ is assumed. The magnitude of $G$ is determined by the Courant number $C_{r}$, the resolution of grid $k \Delta x$, and the dispersion-correction parameter $\gamma$. The stability requirement is $|G| \leq 1$. This requirement is satisfied if $\delta^{2} \leq 1$. The first condition, $\delta \leq 1$, is automatically satisfied, and the second condition, $\delta \geq-1$, gives the following criterion for the Courant number as:

$$
0<C_{r} \leq \sqrt{\frac{-2}{\delta_{1}-\gamma \delta_{2}}}
$$

This criterion gives no explicit relationship between the time step $\Delta t$ and the grid size $\Delta x$, because the $\gamma$ in (22) is also related to $\Delta t$ and $\Delta x$. Figure 3 shows the allowable Courant number $C_{r}$ for given $\gamma$ and $k \Delta x$ to get a stable solution. Judging from Fig. 3 the minimum value of the allowable Courant number occurs when $k \Delta x=\pi$ for $C_{r}>0$. Thus, for the case of $k \Delta x=\pi$ the values $\delta_{1}=-8 / 3$ and $\delta_{2}=64 / 3$ are obtained from (20) and (21), respectively, and (22) gives the stability criterion for the Courant number as:

$$
C_{r} \leq \sqrt{\frac{3}{4+32 \gamma}} .
$$




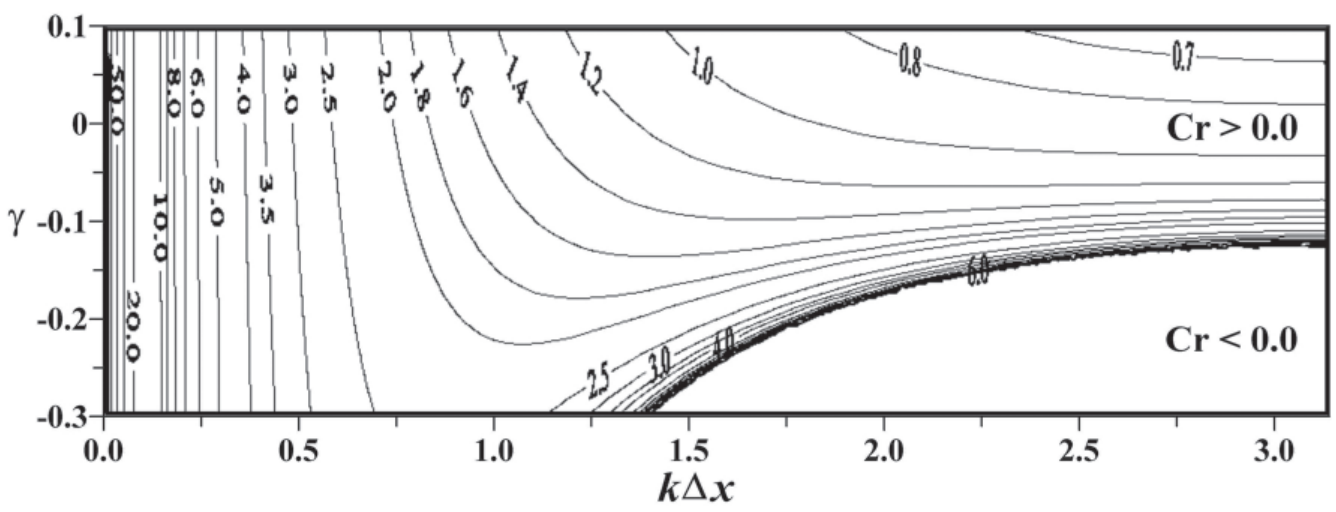

Fig. 3. Allowable $C_{r}$ with respect to $\gamma$ and $k \Delta x$.

The Courant number should be positive as shown in (22). This gives $\gamma \geq \delta_{1} / \delta_{2}(=-0.125)$ for $k \Delta x=\pi$. Another limitation on $\gamma$ for $\Delta x=\infty$ can be found using (17). This gives $\gamma \leq 1 / 12(=0.083)$. As a result, the range of stability for the dispersion-correction parameter when $k \Delta x=\pi$ is theoretically $-0.125 \leq \gamma \leq 0.083$. The most critical value for $C_{r}$ is 0.67 when $\gamma=0.083$. Thus, the stability criterion for the whole range of $k \Delta x$ is $C_{r} \leq 0.67$ with $-0.125 \leq \gamma \leq 0.083$.

The dispersion-correction parameter $\gamma$ calculated by (17) is shown in Fig. 4. This figure shows that the grid size $\Delta x$ greater than $0.63 \Delta x_{\text {Im }}$ must be used for stability because $\Delta x_{\operatorname{Im}} / \Delta x$ is 1.581 for $\gamma=-0.125$. In other words, the uniform grid size greater than approximately 1.27 times of a local water depth must be employed for the stability of the present dispersion-correction scheme.

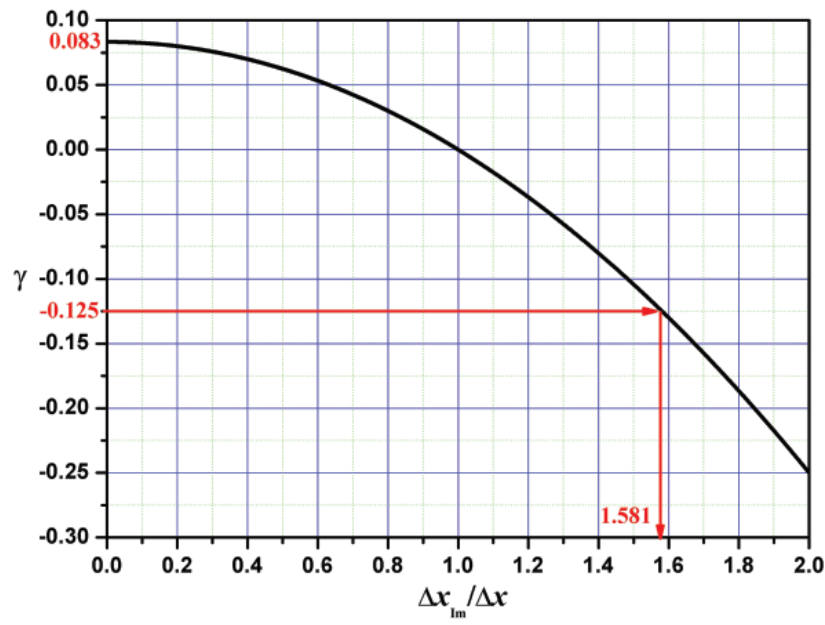

Fig. 4. Dispersion-correction parameter vs. ratio of grid size. 


\subsection{Extension to Slowly Varying Topography}

If the bottom slope changes slowly, it is possible to assume a locally constant water depth at each grid point. Thus, the same dispersion-correction parameter $\gamma$ and the weighting factor $\alpha$ determined for uniform water depth can be employed without loss of generality for the simulation of tsunami propagation over a slowly varying topography. Therefore, if the water depth changes slowly, the linear Boussinesq-type wave equations (5) and (6) can also be solved by employing the numerical scheme developed for constant water depth. The finite difference approximation of (5) with additional shoaling terms for varying topography can be written as:

$$
\begin{aligned}
\frac{v_{i, j}^{n+1 / 2}-v_{i, j}^{n-1 / 2}}{\Delta t} & \left.+g\left(\frac{h_{i+1, j}^{n}-h_{i-1, j}^{n}}{2 \Delta x}\right)\left(\frac{\zeta_{i+1, j}^{n}-\zeta_{i-1, j}^{n}}{2 \Delta x}\right)+\left(\frac{h_{i, j+1}^{n}-h_{i, j-1}^{n}}{2 \Delta y}\right)\left(\frac{\zeta_{i, j+1}^{n}-\zeta_{i, j-1}^{n}}{2 \Delta y}\right)\right] \\
& -g h \mid(1-\alpha)\left(\frac{\zeta_{i+1, j}^{n}-2 \zeta_{i, j}^{n}+\zeta_{i-1, j}^{n}}{\Delta x^{2}}+\frac{\zeta_{i, j+1}^{n}-2 \zeta_{i, j}^{n}+\zeta_{i, j-1}^{n}}{\Delta y^{2}}\right) \\
& +\frac{\alpha}{2}\left(\frac{\zeta_{i+1, j-1}^{n}-2 \zeta_{i, j-1}^{n}+\zeta_{i-1, j-1}^{n}}{\Delta x^{2}}+\frac{\zeta_{i+1, j+1}^{n}-2 \zeta_{i, j+1}^{n}+\zeta_{i-1, j+1}^{n}}{\Delta x^{2}}\right) \\
& +\frac{\alpha}{2}\left(\frac{\zeta_{i-1, j+1}^{n}-2 \zeta_{i-1, j}^{n}+\zeta_{i-1, j-1}^{n}}{\Delta y^{2}}+\frac{\zeta_{i+1, j+1}^{n}-2 \zeta_{i+1, j}^{n}+\zeta_{i+1, j-1}^{n}}{\Delta y^{2}}\right) \mid=0
\end{aligned}
$$

(12) can also be employed for the finite difference representation of (6) by replacing the coefficient $\mathrm{h}^{2} / 3$ by $-\gamma \Delta x^{2}$. (12) is repeated here for completeness:

$$
\frac{\zeta_{i, j}^{n+1}-\zeta_{i, j}^{n}}{\Delta t}-v_{i, j}^{n+1 / 2}+\gamma \Delta x^{2}\left(\frac{v_{i+1, j}^{n+1 / 2}-2 v_{i, j}^{n+1 / 2}+v_{i-1, j}^{n+1 / 2}}{\Delta x^{2}}+\frac{v_{i, j+1}^{n+1 / 2}-2 v_{i, j}^{n+1 / 2}+v_{i, j-1}^{n+1 / 2}}{\Delta y^{2}}\right)=0
$$

where the weighting factor $\alpha$ is given as $1 / 6$ and the dispersion-correction parameter $\gamma$ is given by (17). The validity of the variable depth version of finite difference approximation, i.e., (24) and (25), will be tested in subsequent sections.

\section{TEST OF PRESENT NUMERICAL SCHEME}

\subsection{Tsunami Propagation over Constant Depth Regions}

In order to test the applicability of the present model, the propagation of tsunamis is first simulated with an initial Gaussian shape of the water surface for the case of various constant 
water depths, and the computed free surface displacements are compared with the analytical solutions of the linear Boussinesq equations (Carrier 1991). The initial free surface profile and the velocity of free surface movement are described by (26) and (27), respectively, as:

$$
\begin{aligned}
& \zeta(r, \theta)=2 e^{-(r / a)^{2}}, \\
& \frac{\partial \zeta(r, \theta)}{\partial t}=0,
\end{aligned}
$$

where $a$ is the characteristic radius of the Gaussian function, $r$ [= $\left.\left(x^{2}+y^{2}\right)^{1 / 2}\right]$ denotes the distance from the center of the Gaussian hump, and $\theta$ is the angle from the $x$ axis as shown in Fig. 5.

The analytical solution of the linear Boussinesq equations is given by Carrier (1991) as:

$$
\zeta(r, t)=\int_{0}^{\infty} a^{2} e^{-(k a)^{2} / 4} k \cos \left\lfloor\frac{\sqrt{g h} k t}{\sqrt{1+\frac{(k h)^{2}}{3}}}\right\rfloor J_{0}(k r) d k
$$

where $J_{0}$ is the $0^{\text {th }}$-order Bessel function of the first kind and $g=9.81 \mathrm{~m} \mathrm{~s}^{-2}$. The parameters used for the solution are $a=7500 \mathrm{~m}, \Delta x=2086 \mathrm{~m}$, and $\Delta t=6 \mathrm{sec}$. The numerical simulations using the present scheme are performed for various constant water depths of $h=500,1000$, and $1500 \mathrm{~m}$.
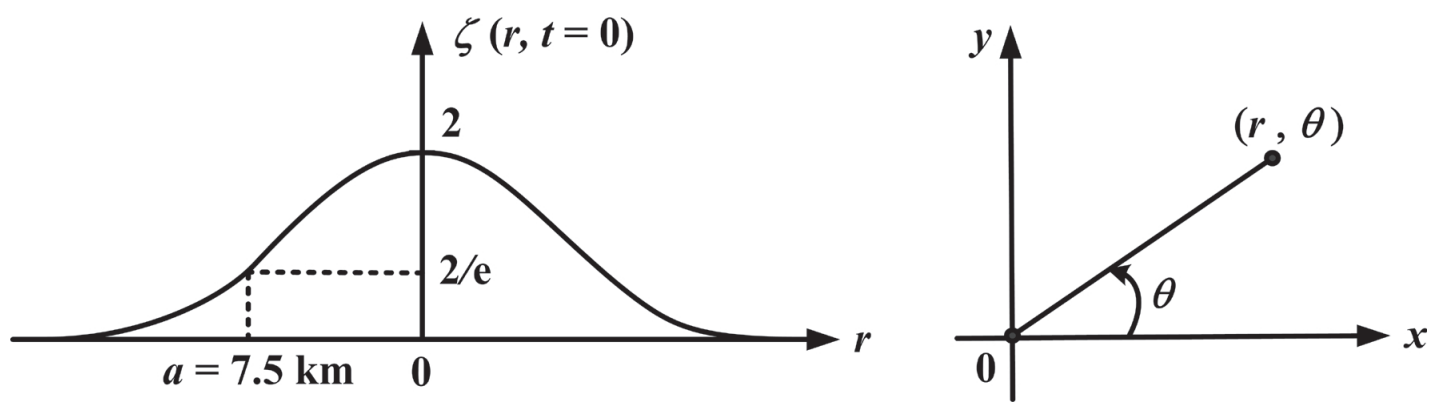

Fig. 5. Coordinate system and initial free surface profile to test the accuracy of the present numerical scheme. 
Figures $6 \sim 8$ present the comparisons of analytical solutions and numerical results calculated considering or neglecting the dispersion-correction parameters $\gamma$ and the weighting factor $\alpha$ proposed in this study. We remark here that the numerical scheme of Imamura et al. (1988) is recovered if we set intentionally $\gamma=\alpha=0.0$. The orientation-free scheme of Cho (1995) is obtained if $\gamma=0.0$ and $\alpha=1 / 6$. These figures show a time history of free surface displacements at the location of $150 \Delta x(r=312900 \mathrm{~m})$ far from the center of the initial Gaussian hump.

The free surface profiles calculated by the present finite difference model considering or neglecting dispersion-correction are compared with the analytical solution of Carrier (1991) for the case of 500-m water depth in Fig. 6. Since the grid size $\Delta x(=2086 \mathrm{~m})$ employed in the computation is larger than $\Delta x_{\mathrm{Im}}(=1085 \mathrm{~m})$ evaluated by (14), the free surface profiles calculated without dispersion-correction are more dispersive than those of the analytical solutions as shown in Fig. 6a. Especially, the numerical model gives a less dispersive profile along the diagonal direction $\left(\theta=45^{\circ}\right)$ than along principal axes $\left(\theta=0^{\circ}, 90^{\circ}\right)$, while the numerical solution with $\alpha=1 / 6$ shows no directional dependency as shown in Fig. $6 \mathrm{~b}$. The present model with the dispersion-correction parameter $\gamma=0.061$ and the weighting factor $\alpha=1 / 6$ gives a good agreement with the analytical solution in the surface profiles in all directions as shown in Fig. 6c. Figure 7 shows the comparison of numerical and analytical free surface profiles for the case of 1000-m water depth. All the numerical solutions except the diagonal profiles calculated with $\alpha=0.0$ agree well with the analytical solutions, because the grid size $\Delta x(=2086 \mathrm{~m})$ coincides with $\Delta x_{\text {Im }}(=2086 \mathrm{~m})$ obtained by $(14)$. On the other hand, for the case of $1500-\mathrm{m}$ water depth, $\Delta x(=2086 \mathrm{~m})$ is smaller than $\Delta x_{\operatorname{Im}}(=3087 \mathrm{~m})$. Thus, the numerical solutions calculated with $\gamma=0.0$ show less dispersive nature in the surface profiles than the analytical solutions based on the linear Boussinesq equations as shown in Figs. 8a and b. The present numerical model using $\gamma=-0.099$ and $\alpha=1 / 6$, however, still gives a correct dispersion effect in each direction as shown in Fig. 8c.

From the results discussed above, it is concluded that the present numerical model is less sensitive to the choice of grid size than the models of Imamura et al. (1988) and Cho (1995). As a result, the present model gives more flexibility in selecting grid size.

\subsection{Tsunami Propagation over a Submerged Circular Shoal}

In this section, the validity of the present finite difference model (FDM) in the case of slowly varying topography is tested using a submerged circular shoal. Figure 9 shows a computational domain with a submerged circular shoal with a conical frustum shape. The center of the shoal is located at $x_{0}=500 \mathrm{~km}$ and $y_{0}=250 \mathrm{~km}$. The base radius $R_{1}$ and the top radius $R_{2}$ are 150 and $86 \mathrm{~km}$, respectively. The bathymetry of the computational domain is given by:

$$
h(r)=\left\{\begin{array}{cc}
1500 & r \geq R_{1} \\
1500 \mathrm{r}^{2} / \mathrm{R}_{1}^{2} & R_{2}<r<R_{1} \\
500 & r \leq R_{2}
\end{array},\right.
$$



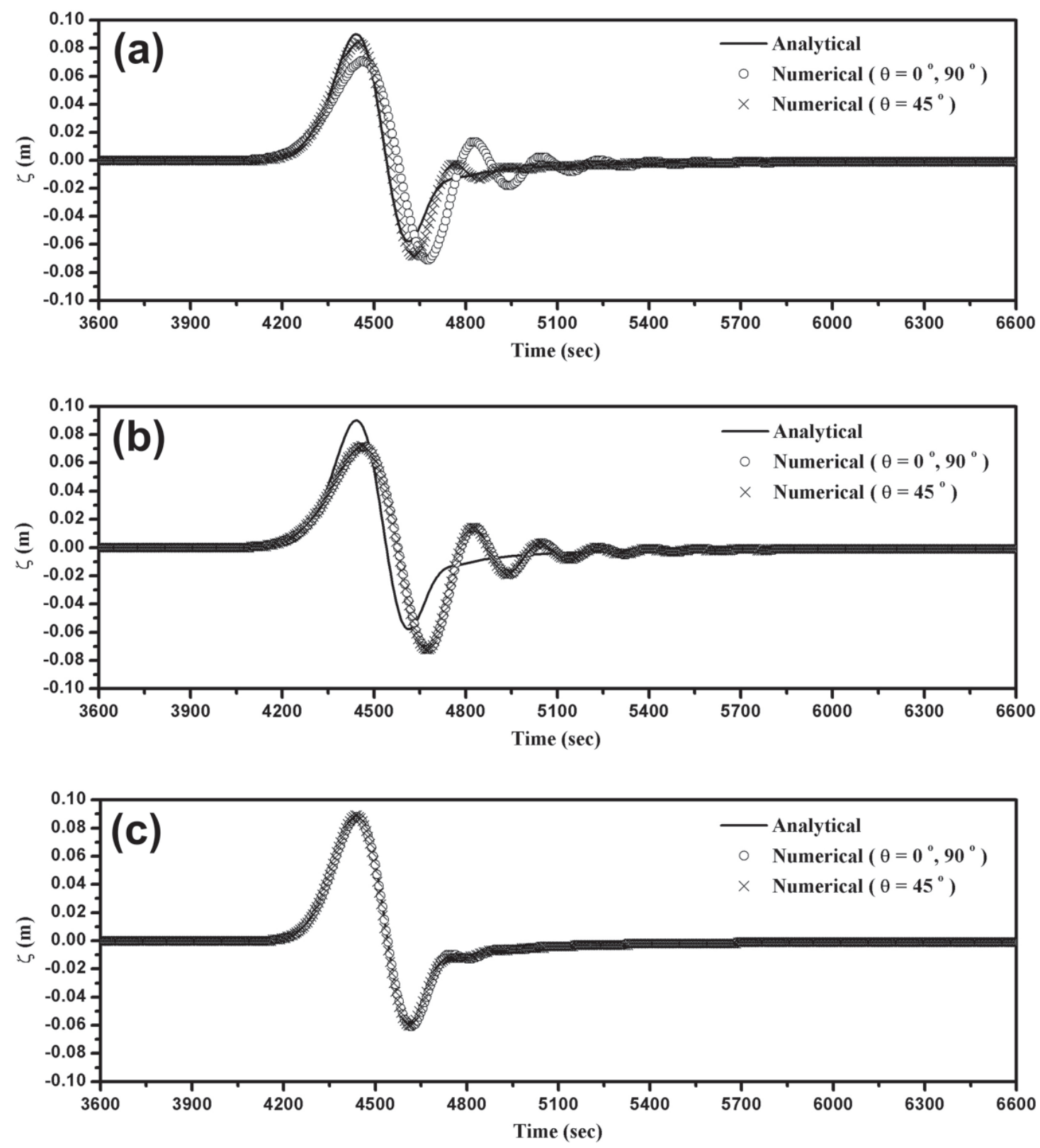

Fig. 6. Comparison of free surface calculated using FDM and analytical solution for the case of $h=500 \mathrm{~m}\left(\Delta x_{\mathrm{Im}}=1085 \mathrm{~m}, \Delta x=2086 \mathrm{~m}\right)$. (a) $\gamma=0.0$, $\alpha=0.0$ (Imamura et al. 1988); (b) $\gamma=0.0, \alpha=1 / 6$ (Cho 1995); (c) $\gamma=0.061$, $\alpha=1 / 6$ (present). 

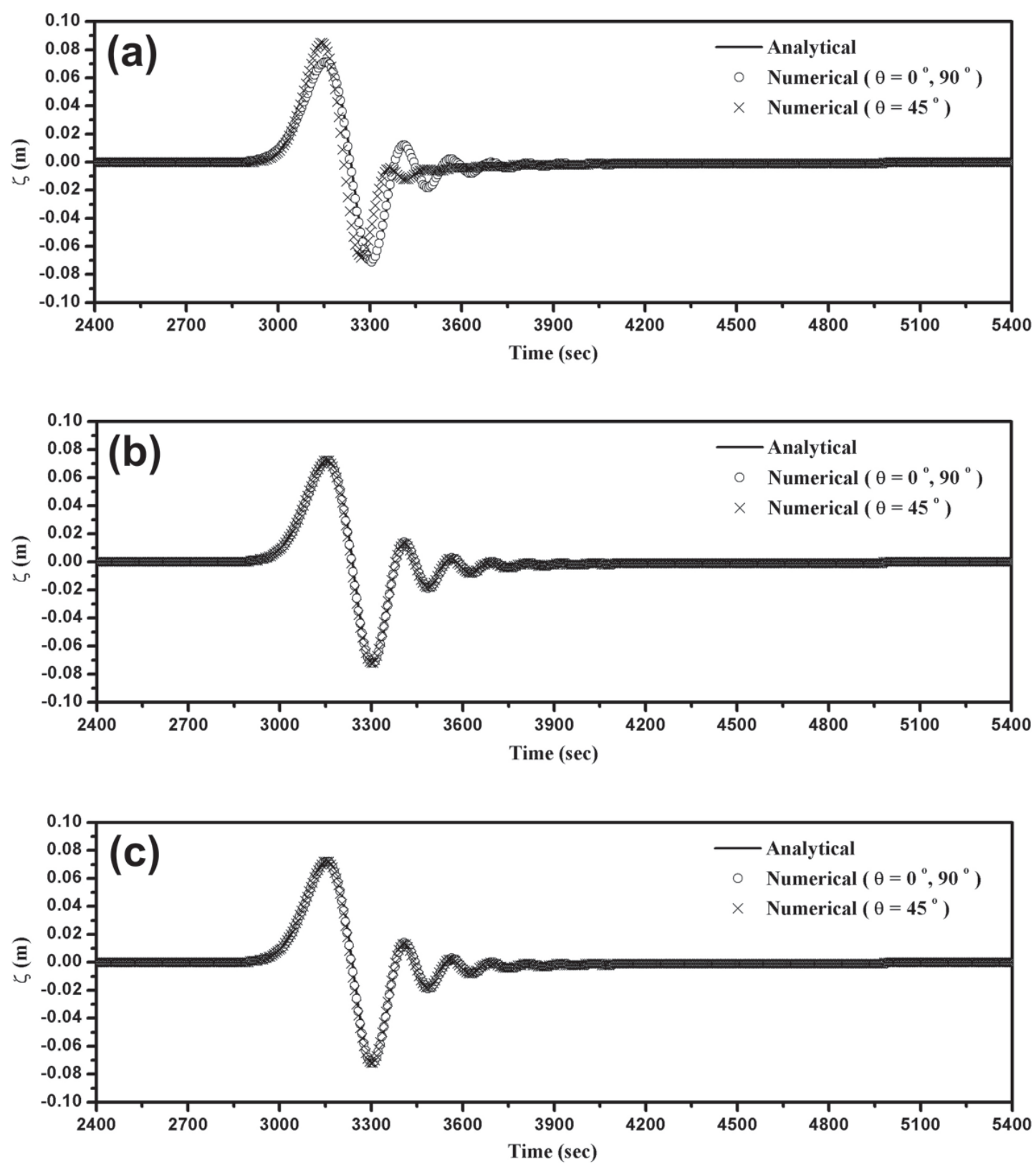

Fig. 7. Comparison of free surface calculated using FDM and analytical solution for the case of $h=1000 \mathrm{~m}\left(\Delta x_{\mathrm{Im}}=2086 \mathrm{~m}, \Delta x=2086 \mathrm{~m}\right)$. (a) $\gamma=0.0$, $\alpha=0.0$ (Imamura et al. 1988); (b) $\gamma=0.0, \alpha=1 / 6$ (Cho 1995); (c) $\gamma=0.0$, $\alpha=1 / 6$ (present). 

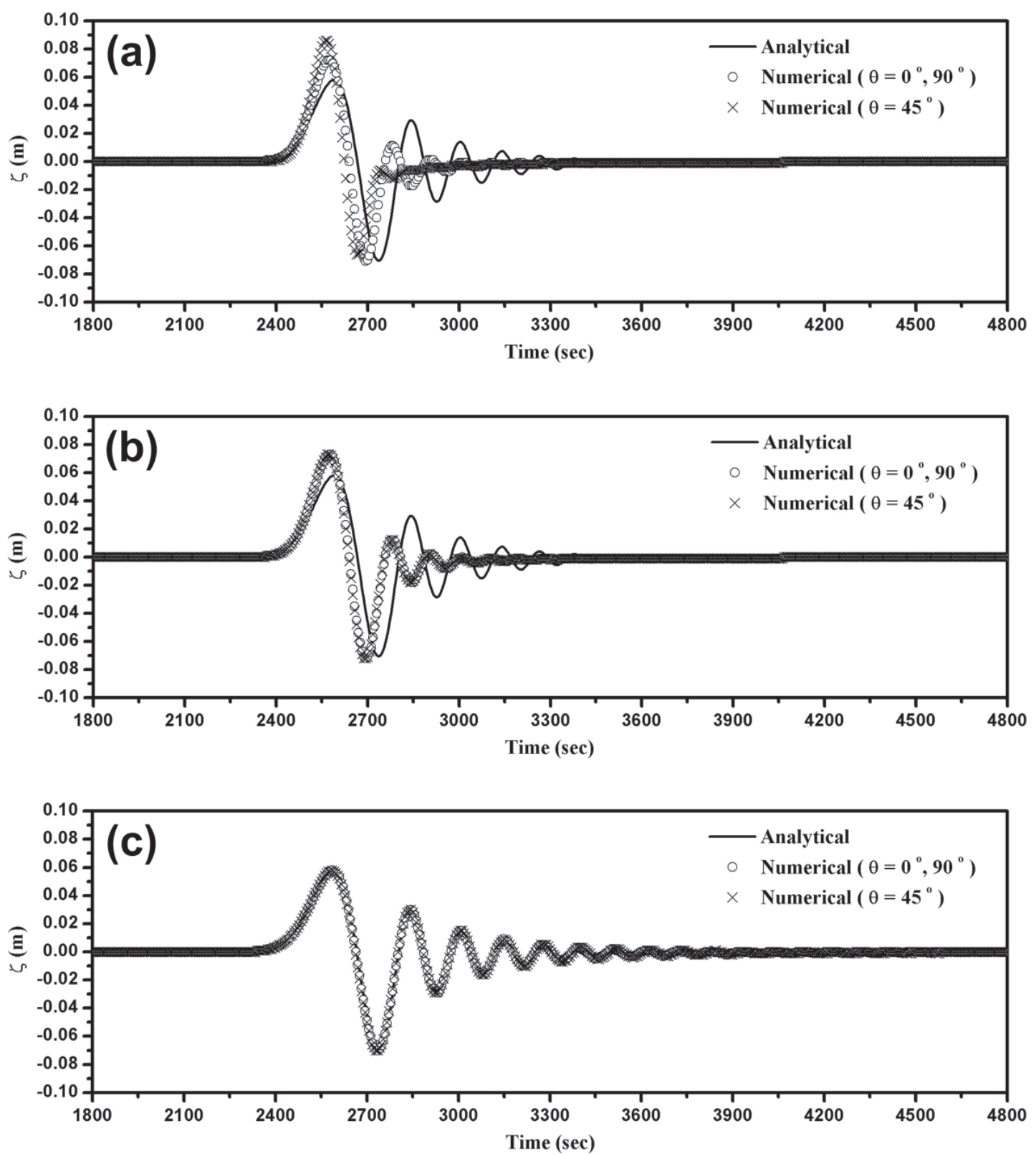

Fig. 8. Comparison of free surface calculated using FDM and analytical solution for the case of $h=1500 \mathrm{~m}\left(\Delta x_{\mathrm{Im}}=3087 \mathrm{~m}, \Delta x=2086 \mathrm{~m}\right)$. (a) $\gamma=0.0$, $\alpha=0.0$ (Imamura et al. 1988); (b) $\gamma=0.0, \alpha=1 / 6$ (Cho 1995); (c) $\gamma=-0.099$, $\alpha=1 / 6$ (present). 


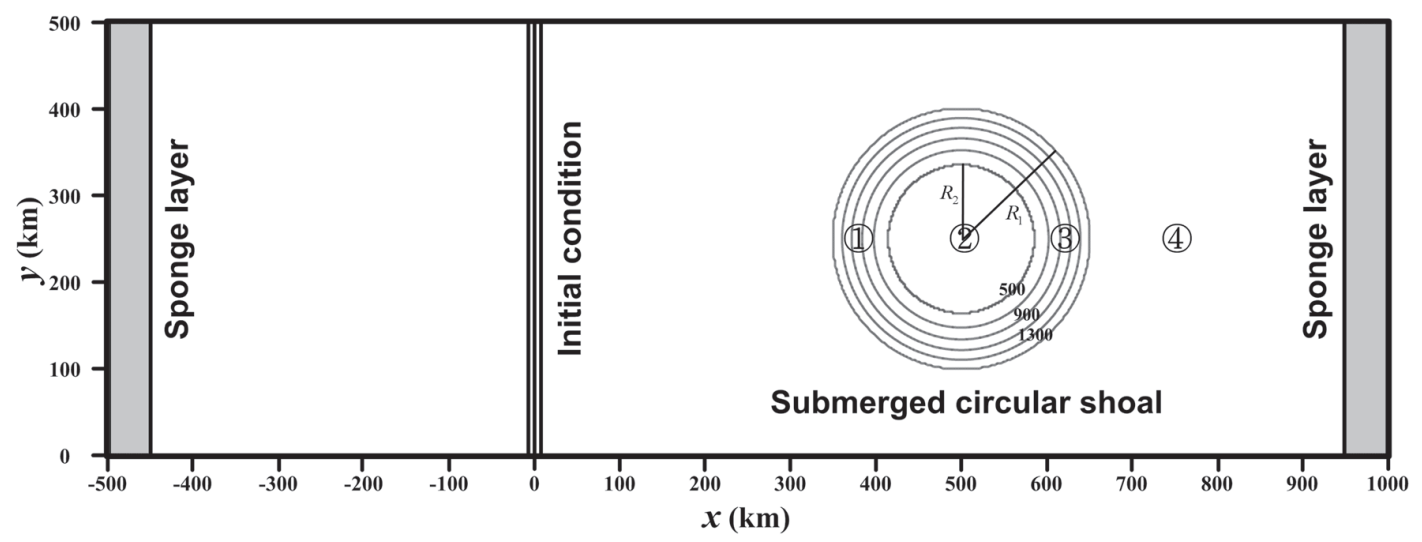

Fig. 9. Schematic diagram of a submerged circular shoal, wave gages and initial condition.

where $r=\sqrt{\left(x-x_{0}\right)^{2}+\left(y-y_{0}\right)^{2}}$ is the distance from the center of the submerged circular shoal.

Along $x=0$, the initial water surface displacement in the form of a solitary wave is prescribed as:

$$
\zeta(x)=2 e^{-(x / 7500)^{2}} .
$$

Four wave gages are installed to measure the free surface profile. Wave gages (1) and (3) are positioned at the front and back slopes of the shoal, respectively, where the water depth is $1000 \mathrm{~m}$. Gage (2) is located at the center of the shoal where the water depth is $500 \mathrm{~m}$. Gage (4) is placed behind the shoal. Sponge layers are placed along both ends of the computational domain to absorb the energy of outgoing waves.

Since no analytical solution is known for this case, numerical solutions using the present dispersion-correction finite difference model are compared with those of the linearized Nwogu's Boussinesq equations (1993) implemented in the FUNWAVE code (Wei and Kirby 1995; Kirby et al. 1998). FUNWAVE is a fully nonlinear Boussinesq wave model with improved dispersion relationships for short waves. The accuracy of FUNWAVE has been verified for various coastal problems such as shoaling, refraction, diffraction and breaking of waves. The numerical simulation using FUNWAVE is performed with a uniform grid size of $500 \mathrm{~m}$, the finest grid allowable on the personal computer employed in this study, to minimize the numerical dispersion. An additional computation using FUNWAVE is also conducted with 2000-m grid size to test the sensitivity of the grid resolution. On the other hand, the numerical simula- 
tion employing the present dispersion-correction model is made with a uniform grid size of $2000 \mathrm{~m}$. The time step $\Delta t$ is determined from the stability criteria for each wave model. The algorithms to compute various physical processes, such as nonlinear advection, nonlinear dispersion and wave dissipation due to bottom friction and breaking, are eliminated from the source code of the FUNWAVE model. Thus, the computational time for the FUNWAVE model to calculate only the propagation step of small-amplitude waves can be measured for fair comparison with that of the present model.

The numerical simulation is conducted for $9000 \mathrm{sec}$ after the initial water surface displacement imposed along $x=0$ is released. The computational time elapsed for different models is presented in Table 1. The FUNWAVE model employing a predictor-corrector scheme consumes a long computational time, while the present fully explicit model takes only $1 / 10$ of the computational time required for FUNWAVE in the case of using the same grid size, i.e., $\Delta x=2000 \mathrm{~m}$. The computational efficiency of the present model can be realized even more dramatically if the computational time is compared with that of FUNWAVE using finer grid size of $\Delta x=500 \mathrm{~m}$. The present model is approximately 2200 times faster than the FUNWAVE model. If the accuracy of the present model is comparable to that of FUNWAVE, it can be concluded that the present model is highly efficient for practical problems.

Figures 10 and 11 are snapshots of the free surface calculated by FUNWAVE and the present FDM, respectively. These figures show clearly that the initial solitary wave evolves into a group of waves due to dispersion of waves as the waves travel a long distance, and also show that the waves are focused behind the shoal due to refraction. Although the grid size for the present model is much larger than that of the FUNWAVE model, the free surface profiles calculated by two different numerical models are almost identical. As shown in Fig. 11a, the present model, however, leaves tiny wiggles on the top of the shoal after the main waves have passed. These wiggles are so called $2 \Delta x$ waves, which appear as a result of strong numerical dispersion caused by the coarse grid employed in the present model. These wiggles can be suppressed if a numerical filter as implemented in FUNWAVE is used.

Table 1. Comparison of computational time for each simulation.

\begin{tabular}{c|c|c|c}
\hline Model & FUNWAVE & FUNWAVE & Present FDM \\
\hline \hline$\Delta x(\mathrm{~m})$ & 500 & 2000 & 2000 \\
Number of grids & $3001 \times 1001$ & $751 \times 251$ & $751 \times 251$ \\
$\Delta t(\mathrm{sec})$ & 2 & 8 & 4 \\
Number of time steps & 4500 & 1125 & 2250 \\
CPU time (sec) & 156888 & 770 & 72 \\
\hline
\end{tabular}



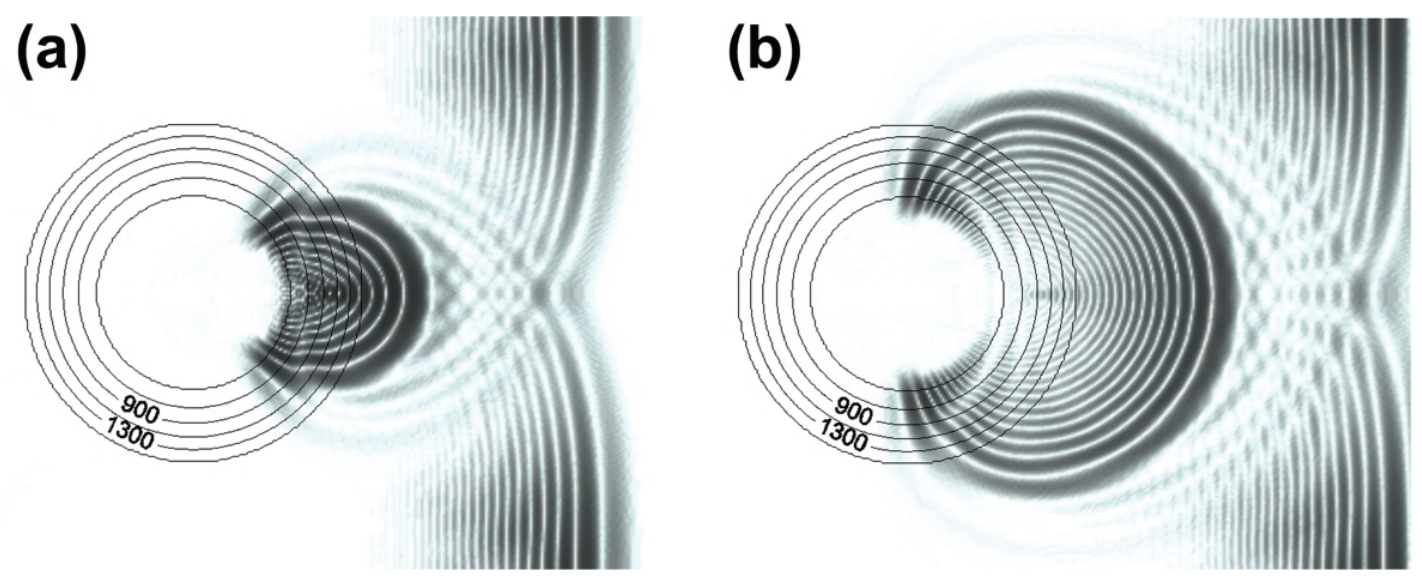

Fig. 10. Snapshots of the free surface computed by FUNWAVE $(\Delta x=500 \mathrm{~m})$. (a) $t=7200 \mathrm{sec}$; (b) $t=8000 \mathrm{sec}$.
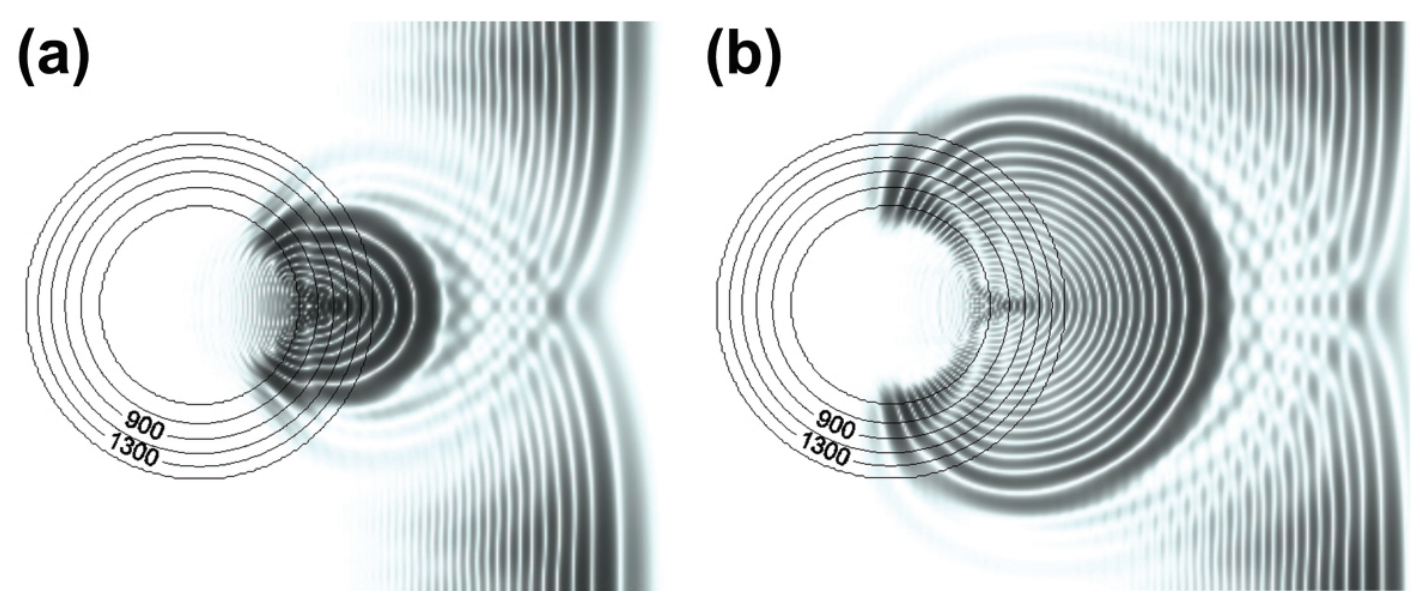

Fig. 11. Snapshots of the free surface computed by present FDM $(\Delta x=2000 \mathrm{~m})$. (a) $t=7200 \mathrm{sec}$; (b) $t=8000 \mathrm{sec}$.

Figure 12 presents the comparison of the free surface time history at various gage locations computed by FUNWAVE and the present FDM. Figure 12a shows the free surface time series recorded at the wave gage (1) located on the front slope of the shoal. This figure shows that the initial solitary wave evolves into a group of waves due to dispersion of waves. Figure 12b 
presents the time series at the location (2) on the top of the shoal. The free surface calculated by the present model shows a good agreement with that of FUNWAVE with a fine grid of $\Delta x=500 \mathrm{~m}$. The results calculated using FUNWAVE with 2000-m grid size, however, show a considerable phase lag in the tail of the wave train. This phase lag is caused by the accumulation of numerical dispersion errors due to poor resolution of computational grid near the top of the shoal. Although the same grid size of $2000 \mathrm{~m}$ is employed, the present model still gives more accurate results. This implies that the present model is capable of minimizing the numerical dispersion efficiently through the dispersion-correction algorithm. Figure $12 \mathrm{c}$ shows the time
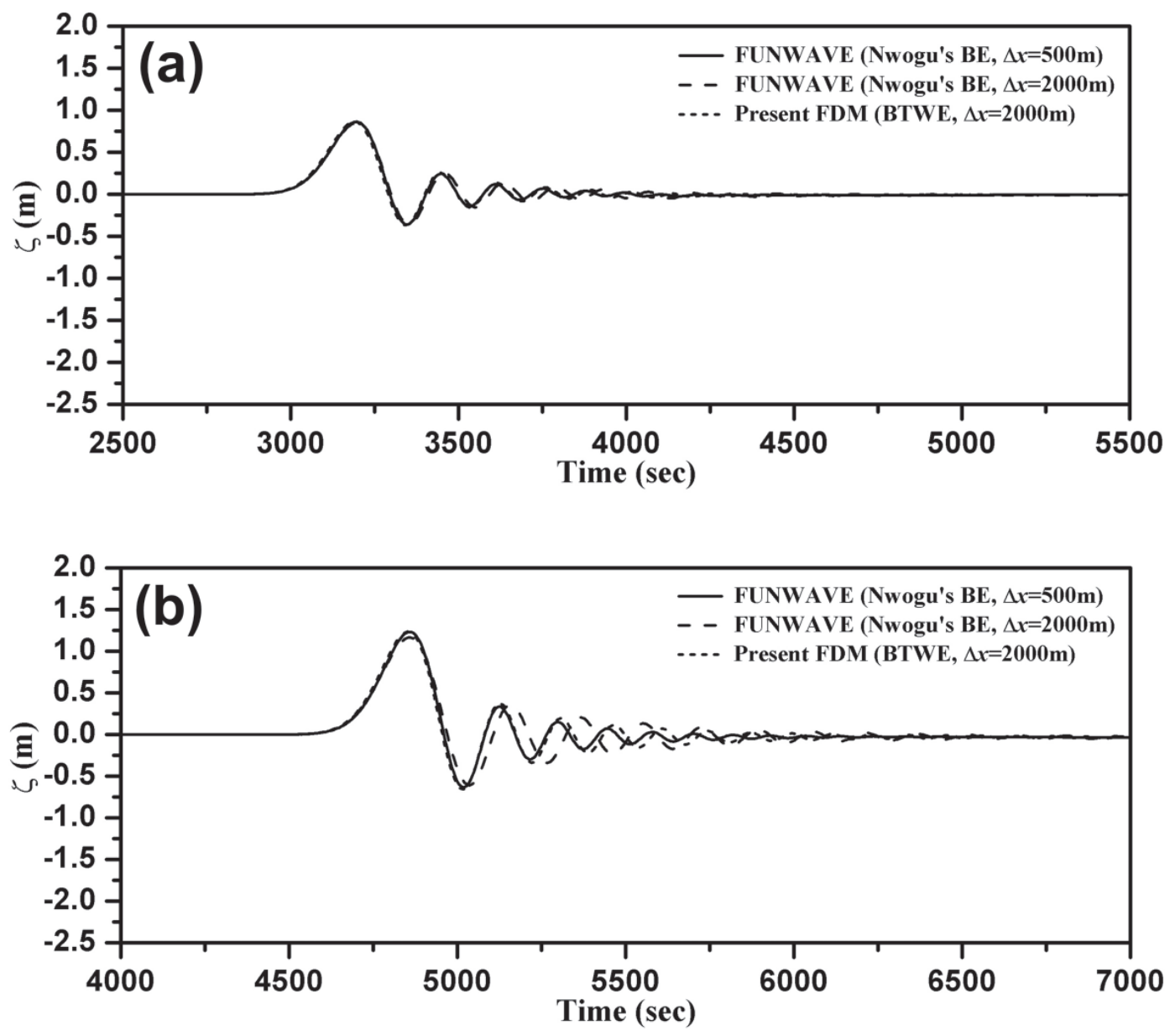

Fig. 12. Comparison of time series computed by FUNWAVE and the present FDM. (a) Time series at location (1) $(h=1000 \mathrm{~m})$; (b) Time series at the location (2) $(h=500 \mathrm{~m})$; (c) Time series at location (3) $(h=1000 \mathrm{~m})$; (d) Time series at location (4) $(h=1500 \mathrm{~m})$. 

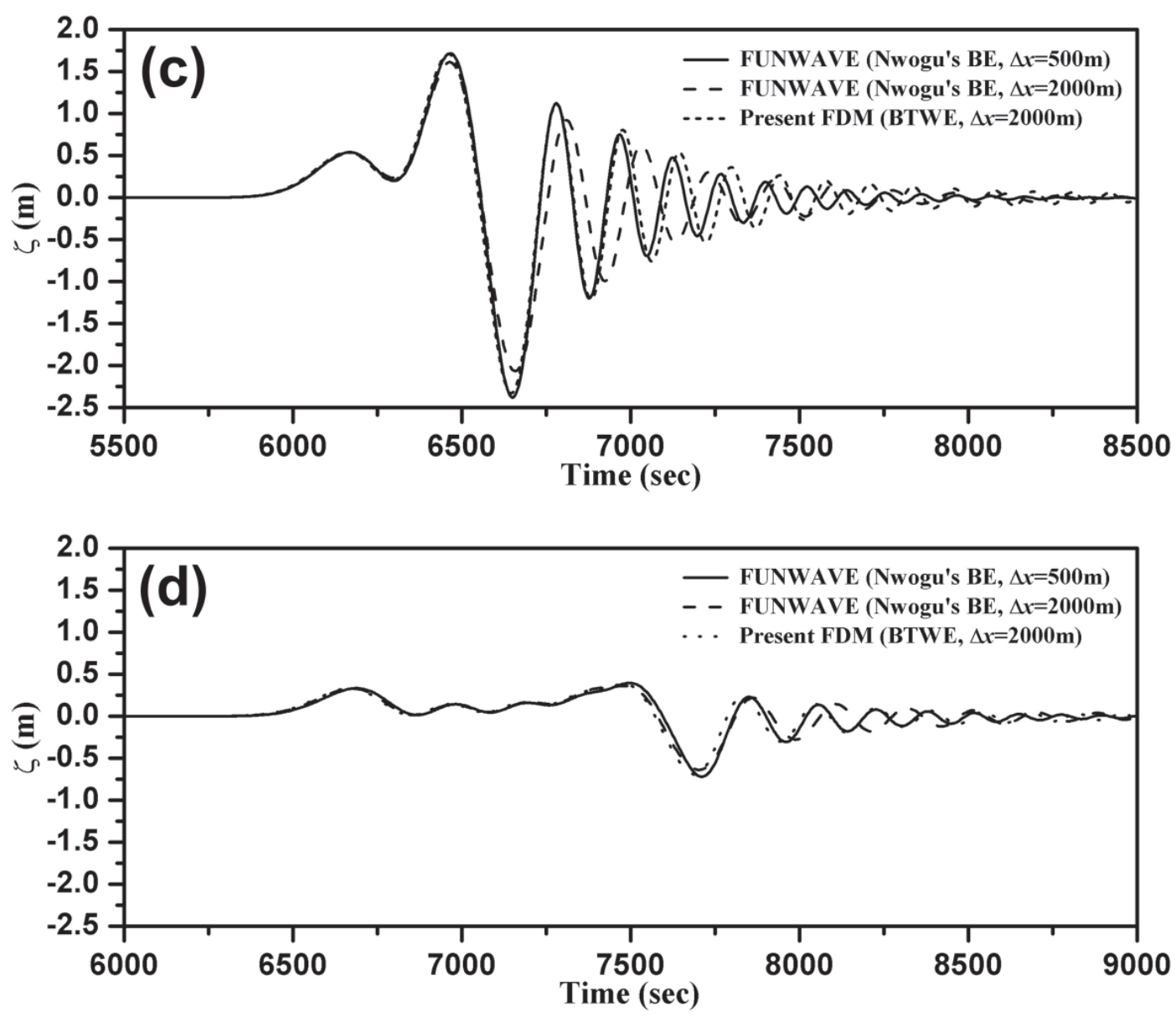

Fig. 12. (Continued)

series at location (3) on the back slope of the shoal. Two trains of waves arrive here. The first train of waves propagating around the shoulders of the shoal meets at this location at $t=6000$ $\mathrm{sec}$. The second train of waves traveling over the top of the shoal arrives here at $t=6300 \mathrm{sec}$. A good agreement between the results calculated using the present model and the FUNWAVE model with fine grids is achieved. The results calculated using FUNWAVE with coarse grids, however, surfers from numerical dispersion. Figure $12 \mathrm{~d}$ shows the time series at location (4) behind the shoal where the water depth $h$ is $1500 \mathrm{~m}$. The agreements between the numerical solutions are reasonable.

In summary, the present FDM is proven to be sufficiently accurate in comparison with the FUNWAVE model, which can deal with a full coverage of dispersion effects for a varying water depth region. On top of this the present model is shown to be highly efficient. Thus, the 
present model can be used as a practical numerical model to simulate the propagation of transoceanic tsunamis over slowly varying topography.

\section{CONCLUSIONS}

An explicit dispersion-correction finite difference model is developed for the simulation of far-field tsunami propagation. The present model employs a linear Boussinesq-type wave equation. The present model can suppress the numerical dispersion arising from the explicit scheme and take into account the physical dispersion of waves. The dispersion-correction scheme was tested for the case of the propagation of Gaussian hump over various constant water depths. Comparisons of the results with the analytical solution of the linear Boussinesq equations are made. Even though the simulations are performed using a fixed grid size over various constant water depths, the results using the proposed dispersion-correction scheme agree well with the analytical solution. In order to test the present numerical model for varying water depth a simulation of the solitary wave of Gaussian shape propagating over a submerged circular shoal was conducted and the results were compared with the numerical solutions of the linearized Nwogu's Boussinesq equations implemented in the FUNWAVE model. The numerical results show that the present model gives a sufficiently accurate solution comparable to the result obtained by the models employing Boussinesq equations. Moreover, the present model is shown to be highly efficient. Thus, the present model can be used as a practical alternative to the numerical models based on the full Boussinesq equations to simulate the propagation of distant tsunamis over slowly varying topography. The present model, however, has some limitations, i.e., the grid size has to be equal in both horizontal directions and the slope of the bottom should be slowly varying.

Acknowledgements This research was supported by a grant (No. NEMA-06-NH-06) from the Natural Hazard Mitigation Research Group, National Emergency Management Agency of Korea.

\section{REFERENCES}

Abbott, M. B., A. D. McCowan, and I. R. Warren, 1981: Numerical modeling of free-surface flows that are two-dimensional in plan. In: Fisher, H. B. (Ed.), Transport Models for Inland and Coastal Waters, Academic Press, 222-283.

Carrier, G. F., 1991: Tsunami propagation from a finite source. Proc. of $2^{\text {nd }}$ UJNR Tsunami Workshop, NGDC, Hawaii, 101-115.

Cho, Y. S., 1995: Numerical simulations of tsunami propagation and run-up. Ph. D. Thesis, Cornell University, New York, USA, 264 pp.

Goto, C., and N. Shuto, 1983: Numerical simulation of tsunami propagations and run-up. In: Iida, K. and T. Iwasaki (Eds.), Tsunami: Their Science and Engineering, Terra Science, Tokyo, 439-451. 
Hsiao, S. C., P. Lynett, H. H. Hwung, and P. L. F. Liu, 2005: Numerical simulations of nonlinear short waves using the multi-layer model. J. Eng. Mech., ASCE, 131, 231-243.

Hwang, L. S., and D. Divoky, 1970: Tsunami generation. J. Geophys. Res., 75, 6802-6817.

Imamura, F., N. Shuto, and C. Goto, 1988: Numerical simulation of the transoceanic propagation of tsunamis. Proc. of $6^{\text {th }}$ Congress Asian and Pacific Regional Division, IAHR, Japan, 265-271.

Kirby, J. T., G. Wei, Q. Chen, A. B. Kennedy, and R. A. Dalrymple, 1998: Fully nonlinear Boussinesq wave model. User Manual, Rep. No. CACR-98-06, Univ. of Delaware.

Kowalik, Z., and T. S. Murty, 1984: Computation of tsunami amplitudes resulting from a predicted major earthquake in the Shumagin seismic gap. Geophys. Res. Lett., 11, 12431246.

Krenk, S., 2001: Dispersion-corrected explicit integration of the wave equation. Comp. Meth. Appl. Mech. Eng., 191, 975-987.

Lapidus, L., and G. F. Pinder, 1982: Numerical solution of partial differential equations in science and engineering, John Wiley \& Sons.

Lynett, P., and P. L. F. Liu, 2004: A two-layer approach to water wave modeling. Proc. $R$. Soc. Lond. A, 460, 2637-2669.

Mader, C. L., and G. D. Curtis, 1991: Numerical modeling of tsunami inundation of Hilo harbor. JIMAR Contribution No. 91-251, University of Hawaii, Honolulu.

Nwogu, O., 1993: Alternative form of Boussinesq equations for nearshore wave propagation. J. Waterw. Port Coast. Ocean Eng., ASCE, 119, 618-638.

Wei, G., and J. T. Kirby, 1995: A time-dependent numerical code for the extended Boussinesq equations. J. Waterw. Port Coast. Ocean Eng., ASCE, 121, 251-261.

Yoon, S. B., 2002: Propagation of distant tsunamis over slowly varying topography. J. Geophys. Res., 107, doi:10.1029/2001JC000791.

Yoon, S. B., C. H. Lim, and J. Choi, 2007: Dispersion-correction finite difference model for simulation of transoceanic tsunamis. Terr. Atmos. Ocean. Sci., 18, 31-53. 